\title{
On Generalized Localization of Fourier Inversion Associated with an Elliptic Operator for Distributions
}

\author{
Ravshan Ashurov, ${ }^{1}$ Almaz Butaev ${ }^{2,3}$ and Biswajeet Pradhan ${ }^{2,3}$ \\ ${ }^{1}$ Institute of Mathematics, National University of Uzbekistan, Hodjaeva Street 29, \\ Tashkent 100125, Uzbekistan \\ ${ }^{2}$ Institute of Advanced Technology (ITMA), University Putra Malaysia, 43400 Serdang, \\ Selangor, Malaysia \\ ${ }^{3}$ Department of Civil Engineering, Faculty of Engineering, Geospatial, Information Science \\ Center (GIS RC), University Putra Malaysia (UPM), 43400 Serdang, Selangor, Malaysia
}

Correspondence should be addressed to Biswajeet Pradhan, biswajeet@putra.upm.edu.my

Received 24 May 2012; Accepted 29 June 2012

Academic Editor: Allaberen Ashyralyev

Copyright (C) 2012 Ravshan Ashurov et al. This is an open access article distributed under the Creative Commons Attribution License, which permits unrestricted use, distribution, and reproduction in any medium, provided the original work is properly cited.

We study the behavior of Fourier integrals summed by the symbols of elliptic operators and pointwise convergence of Fourier inversion. We consider generalized localization principle which in classical $L_{p}$ spaces was investigated by Sjölin $(1983)$, Carbery and Soria $(1988,1997)$ and Alimov (1993). Proceeding these studies, in this paper, we establish sharp conditions for generalized localization in the class of finitely supported distributions.

\section{Introduction}

In this paper, we study the behavior of spherical Fourier integrals and pointwise convergence and summability of Fourier inversion.

Let

$$
A(D)=\sum_{|\alpha|=m} c_{\alpha} D^{\alpha}
$$

be a homogeneous elliptic differential operator of order $m$. Let us consider its symbol defined as polynomial:

$$
A(x)=\sum_{|\alpha|=m} c_{\alpha} x^{\alpha}
$$


and assume that the Gaussian curvature of surface $S=\left\{x \in R^{n}: A(x)=1\right\}$ is always strictly positive.

We recall that for $f \in L_{2}\left(R^{n}\right)$ its Fourier transform is defined as

$$
\widehat{f}(\xi)=\int f(y) e^{-i y \xi} d y
$$

and partial Fourier integral associated with elliptic operator (1.1):

$$
E_{\curlywedge} f(x)=(2 \pi)^{-n} \int_{A(\xi)<\curlywedge} \hat{f}(\xi) d \xi
$$

(note that throughout the paper we consider only Lebesque measure on $R^{n}$ and $\int=\int_{R^{n}}$ ). For some functions, Fourier integrals do not converge pointwisely and various summation techniques are applied to recover convergence property. In this paper, we consider the method of the Riesz means. The Riesz means of order $s$ are defined as

$$
E_{\lambda}^{s} f(x)=(2 \pi)^{-n} \int_{A(\xi) \leq \Lambda}\left(1-\frac{|\xi|^{2}}{\lambda^{2}}\right)^{s} \widehat{f}(\xi) e^{i \xi x} d \xi
$$

As an example, one can consider Laplacian $A(D)=\sum_{i=1}^{n}\left(\partial^{2} / \partial x_{i}^{2}\right)$, and note that the level surfaces of its symbol are Euclidean spheres. Thus, Fourier inversion associated with Laplace operator has the form:

$$
E_{\lambda} f(x)=(2 \pi)^{-n} \int_{|\xi|^{2}<\lambda} \widehat{f}(\xi) e^{i \xi x} d \xi
$$

and known as spherical partial Fourier integrals. The question of $E_{\lambda} f(x)$ convergence to $f(x)$ almost everywhere is not solved in $R^{n}, n \geq 2$ even for classical $L_{2}$ functions and presents one of the most challenging open problems of classical harmonic analysis, and even special cases of this problem are of particular interest. One of such special cases is the problem of generalized localization, which for the first time was formulated by V. Ii'in in [1]. For convenience, we give its definition for the Riesz means $E_{\lambda}^{s}$.

Definition 1.1. We say that, for the Riesz means of order $s$, the generalized localization principle in function class $\mathfrak{F}$ is satisfied, if for any function $f \in \mathfrak{F}$, the equality

$$
\lim _{\curlywedge \rightarrow \infty} E_{\lambda}^{s} f(x)=0
$$

is true for a.e. $x \in R^{n} \backslash \operatorname{supp} f$.

This localization principle generalizes the classical Riemann localization principle and for $L_{p}$ functions was intensively investigated by Sjölin [2], Carbery and Soria [3, 4], Bastis [5-7], and Ashurov et al. [8]. It was established that $R^{n}$ localization holds true in $L_{p}$, where $p \in[2,2 n /(n-1)]$ and fails otherwise. 
Over the last several years, a number of Fourier inversion studies considered distributions and investigated the behavior of their Fourier integrals (see, e.g., [9-12]). In particular, Alimov in [13] considered the classical Riemann localization principle for compactly supported distributions and established criteria for its validity (see also [14, 15]).

In this paper, we study generalized localization principle for compactly supported distributions and present conditions for its fulfillment.

\section{Notation and Definitions}

We define Schwartz space $S\left(R^{n}\right)$ as the function class of all infinitely differentiable functions that are rapidly decreasing at infinity along with all partial derivatives. It is well known that $S\left(R^{n}\right)$, being equipped with a family of seminorms

$$
d_{\alpha, \beta}(\phi)=\sup _{x \in R^{n}}\left|x^{\alpha} D^{\beta} \phi(x)\right|
$$

is a Frechet space (here $\alpha, \beta$ are multi-indices and $D$ is a partial derivative). As usual, we also consider class of tempered distributions $S^{\prime}$ defined as dual to $S$.

Let $\varepsilon$ be the space of infinitely differentiable functions with topology $\tau_{E}$ such that $\phi_{n} \rightarrow$ 0 in $\tau_{E}$ if and only if for each multiindex $\alpha$ and compact $K$

$$
\sup _{x \in K} D^{\alpha} \phi_{n}(x) \longrightarrow 0
$$

As usual we denote its conjugate space by $\mathcal{E}^{\prime}$.

It is known (see, e.g., [16]) that each $f \in \mathcal{E}^{\prime}$ has finite support and equivalent to the class of finitely supported tempered distributions. Thus, it follows from the Paley-Wienner theorem that, for each $f \in \mathcal{E}^{\prime}$, its Fourier transform $\widehat{f} \in C^{\infty}$. Since $\widehat{f}$ is locally integrable, it is natural to define Fourier integral of $f \in \mathcal{E}^{\prime}$ and its Riesz means by (1.4) and (1.5), respectively. operator:

We also note that for $f \in L_{2}$ the Riesz mean $E_{\lambda}^{s} f$ can be considered as an integral

$$
E_{\lambda}^{s} f(x)=(2 \pi)^{-n} \int f(y) \theta_{\lambda}^{s}(x-y) d y
$$

with kernel $\theta_{\lambda}^{s}(y)=\widehat{m}_{\lambda}^{s}(y)$ where

$$
m_{\lambda}^{S}(y)=\left(1-\frac{A(y)}{\lambda}\right)_{+}^{s}
$$

where $(1-A(y) / \lambda)_{+}^{S}=(1-A(y) / \lambda)^{S} \cdot \chi_{A(y)<\lambda}(y)$. 
Representation (2.3) has its natural analogue for $f \in \mathcal{E}^{\prime}$. Let $\psi_{n}$ be a sequence of Schwartz functions such that $\psi_{n}(y)=0$ as $|y|>\lambda$ and $\psi_{n}(y) \rightarrow m_{\lambda}^{s}(y)$ in $L_{1}$ norm. Then:

$$
\begin{aligned}
E_{\lambda}^{S} f(x) & =\lim _{n \rightarrow \infty}(2 \pi)^{-n} \int \widehat{f}(\xi) \psi_{n}(\xi) e^{i x \xi} d \xi \\
& =(2 \pi)^{-n} \lim _{n \rightarrow \infty}\left\langle\widehat{f}(\xi), \psi_{n}(\xi) e^{i x \xi}\right\rangle \\
& =(2 \pi)^{-n} \lim _{n \rightarrow \infty}\left\langle f(y), \widehat{\psi}_{n}(x-y)\right\rangle .
\end{aligned}
$$

Note that inequality $\|\widehat{g}\|_{\infty} \leq\|g\|_{1}$ implies that $\widehat{\psi}_{n} \rightarrow \widehat{m}_{\lambda}^{s}$ in $\mathcal{E}$ and since $f$ is continuous on $\mathcal{\varepsilon}$

$$
E_{\lambda}^{s} f(x)=(2 \pi)^{-n}\left\langle f(\cdot), \theta_{\lambda}^{s}(x-\cdot)\right\rangle .
$$

We will need Sobolev's classes which can be defined for $l \in \mathbb{R}$ in the following way.

Definition 2.1. We say that tempered distribution $f$ belongs to Sobolev class $H^{l}$ if $\widehat{f}$ is a regular distribution such that

$$
\|f\|_{H^{l}}^{2}=\int|\widehat{f}(\xi)|^{2}\left(1+|\xi|^{2}\right)^{l} d \xi<\infty
$$

One can see that, in particular, $H^{0}=L_{2}$. We also remark that for every $f \in \mathcal{E}^{\prime}$ there is $l \in \mathbb{R}$ such that $f \in H^{l}$ (for proof see, e.g., [16]).

In other respects, we make the following conventions:

(i) symbol $J_{v}$ is used to denote Bessel function of the first kind and order $v \geq 0$,

(ii) $\chi_{E}$ is preserved for an indicator function of $E \subset R^{n}$,

(iii) unless otherwise indicated, all functions are assumed to be defined on $R^{n}$ and by definition $L_{p}(\Omega) \equiv\left\{f \in L_{p}\left(R^{n}\right): \operatorname{supp} f \subset \Omega \subset R^{n}\right\}$.

\section{Main Result}

As has been mentioned above, every $f \in \mathcal{E}^{\prime}$ belongs to some Sobolev classes $H^{l}$, in this paper, we use this fact to establish criterion of generalized localization for finitely supported distributions. The following theorems present major results of current study.

Theorem 3.1. Let $f \in \mathcal{E}^{\prime} \cap H^{-l}, l \geq 0$. Then, for integer $s \geq l$, equality

$$
\lim _{\lambda \rightarrow \infty} E_{\lambda}^{s} f(x)=0
$$

holds true a.e. on $R^{n} \backslash \operatorname{supp} f$.

Our approach is based on the methods by Carbery and Soria [3] and in order to prove Theorem 3.1, we will follow his idea first proving some auxiliary facts in the following section. 


\section{Dual Sets}

Let $a(x)=[A(x)]^{1 / m}$ and $K=\left\{x \in R^{n}: a(x) \leq 1\right\}$. Then, $K$ is a symmetric body that is convex compact symmetric set. We recall that set $K^{*}=\{y:|x \cdot y| \leq 1, \forall x \in K\}$ is called polar set with respect to $K$.

As it is done in [17], we will introduce the norm $\|\cdot\|_{a}$ generated by $a(x)$ as

$$
\|x\|_{a}=a(x)
$$

and dual norm $\|\cdot\|_{a}^{*}$ as

$$
\|y\|_{a}^{*}=\sup _{\|x\|_{a} \leq 1}|x \cdot y|=\sup _{\|x\|_{a}=1}|x \cdot y|
$$

Next, let $S$ and $S^{*}$ be the boundaries of $K$ and $K^{*}$, respectively.

It is not difficult to show that $S^{*}=\{\nabla a(x), x \in S\}$. Indeed on the one hand $a(\lambda x)=$ $\lambda a(x)$ and, therefore, for $x \in S$

$$
\begin{gathered}
x \cdot \nabla a(x)=\frac{d a}{d r}(x)=a(x)=1, \\
(-x) \cdot \nabla a(x)=-\frac{d a}{d r}(x)=a(x)=-1,
\end{gathered}
$$

which means that $\|\nabla a(x)\|_{a}^{*} \geq 1$. On the other hand, for any $y \in S$, one can consider $F(y)=$ $y \cdot \nabla a(x)$ and examine its local extremums on the surface $S$. Since $S$ is compact, $F(y)$ reaches its extremum values and it is known that, at extremum points, $\nabla F(y)$ must be parallel to the normal to $S$ at point $y$, which is parallel to $\nabla a(y)$. Since $\nabla F(y)=\nabla a(x)$, we can conclude that $\nabla a(x) \| \nabla a(y)$ at the extremum points. Since $S$ is strictly convex, it is possible only for $y= \pm x$, that implies $\|\nabla a(x)\|_{a}^{*} \leq 1$.

It is convenient for given $x \in R^{n}$ to use the notation $\theta(x)$ to denote the point on $S$ such that the outer normal to $S$ at $\theta(x)$ is parallel to $x$. Similarly, we denote $\eta(x)$ the point on $S^{*}$ such that the outer normal to $S^{*}$ at $\eta(x)$ is parallel to $x$. One can remark that we have just seen that for $y \in S^{*}$

$$
y \cdot \theta(y)=1
$$

\section{Technical Lemmas for Theorem 3.1}

We will need the asymptotic representation of $\theta_{\lambda}^{s}(y)$, which can be derived by stationaryphase method (see, e.g., [18]):

$$
\theta_{\lambda}^{s}(y)=\lambda^{(n-1) / 2}|y|^{-(n+1) / 2} \cdot\left[R_{+}^{s}(y, \lambda) e^{i \lambda y \cdot \theta(y)}+R_{-}^{s}(y, \lambda) e^{-i \lambda y \cdot \theta(y)}\right]
$$


where functions $R_{ \pm}^{s}(y, \lambda) \in C^{\infty}(\{y:|y|>\epsilon\} \times[1, \infty))$ and

$$
D_{y}^{\alpha} D_{\lambda}^{\beta} R_{ \pm}^{s}(y, \lambda)=O\left(\lambda^{-s-\beta}\right)
$$

uniformly on $|y|>\delta$ and $\lambda>\delta$.

Now, let us consider positive numbers $\varepsilon$ and $R, \varepsilon<R$ and function $\phi(x)=\phi\left(\|x\|_{a}^{*}\right) \in$ $C_{0}^{\infty}$ vanishing on $\left\{x:\left(\|x\|_{a}^{*}<\varepsilon\right) \vee\left(\|x\|_{a}^{*}>R\right)\right\}$. Then, for $s \geq 0$, we set by definition

$$
\Theta_{\lambda}^{s}(x)=\phi(x) \theta_{\lambda}^{s}(x),
$$

where $\theta_{\lambda}^{s}$ as in (2.3).

We will need some estimates for the Fourier transform of $\Theta_{\lambda}^{s}$. With this aim, we will need the following lemmas.

Lemma 5.1. Let $t \geq \delta>0$ and $|\xi|<1$. Then, for any $\alpha>0$

$$
\left|\widehat{\Theta}_{t}^{s}(\xi)\right| \leq O\left(t^{-\alpha}\right)
$$

Proof. This estimate easily follows from the definition of $\Theta_{t}^{s}$. Indeed,

$$
\begin{aligned}
\widehat{\Theta}_{t}^{s}(\xi)= & \int \theta_{t}^{s}(x) \phi(x) e^{-i \xi x} d x=\int\left(1-\widehat{\frac{A(y)}{t}}\right)_{+}^{s}(x) \phi(x) e^{-i \xi x} d x \\
= & \int\left(1-\frac{A(x)}{t}\right)_{+}^{s} \widehat{\phi}(x+\xi) d x=\int_{A(x)<t} \widehat{\phi}(x+\xi) d x \\
& +\sum_{k=1}^{s} \frac{C_{k}}{t^{k}} \int_{A(x)<t} \widehat{\phi_{k}(\cdot, \xi)}(x) d x
\end{aligned}
$$

where $\phi_{k}(y, \xi)=B^{k}\left(D_{y}\right)\left[\phi(y) e^{-i y \xi}\right]$ and $B(D)$ is formally conjugate to operator $A(D)$. Since $\phi(0)=\phi_{k}(0, \xi)=0$,

$$
\widehat{\Theta}_{t}^{s}(\xi)=\int_{A(x)>t} \widehat{\phi}(x+\xi) d x+\sum_{k=1}^{s} \frac{C_{k}}{t^{k}} \int_{A(x)>t} \widehat{\phi_{k}(\cdot, \xi)}(x) d x
$$

Further, we notice that since $\phi_{k}(y, \xi) \in C_{0}^{\infty}$ then for any $\alpha>0$ there is $C_{\alpha}$ such that functions $\widehat{\phi}_{k}(x, \xi)=C_{\alpha} /(1+A(x))^{\alpha}$, uniformly for $k=1, \ldots, s$ and $|\xi|<1$. For the same reason, for any $\alpha>0$, one has $\widehat{\phi}(x) \leq O\left((1+x)^{-\alpha}\right)$. Now substituting these estimates into (5.6), we complete the proof.

Lemma 5.2. Let $t \geq \delta>0$ and $|\xi| \geq 1$. Then, for any $\alpha>0$,

$$
\left|\widehat{\Theta}_{t}^{s}(\xi)\right|=\frac{O(1) t^{-s}}{\left(1+\left|\|\xi\|_{a}-t\right|\right)^{\alpha}} .
$$


Proof. By definition,

$$
\widehat{\Theta}_{t}^{s}(\xi)=\int_{\epsilon<\|y\|_{a}^{*}<R} \phi(y) \theta_{t}^{s}(y) e^{-i \xi \cdot y} d y
$$

Let us pass to a new coordinate system $y \rightarrow\left(r=\|y\|_{a}^{*}, \eta=\eta(y)\right)$. Then,

$$
\widehat{\Theta}_{t}^{s}(\xi)=\int_{\epsilon}^{R} \phi(r) r^{n-1} \int_{\eta \in S^{*}} \theta_{t}^{s}(r \eta) e^{-i r \xi \eta \eta} d \sigma(\eta) d r,
$$

where $d \sigma(\eta)$ is a Lebesgue surface measure of $S^{*}$.

Using (5.1), we have

$$
\begin{aligned}
\widehat{\Theta}_{t}^{S}(\xi)= & t^{(n-1) / 2} \int_{\epsilon}^{R} \phi(r) r^{(n-3) / 2} \int_{\eta \in S^{*}}|\eta|^{-(n+1) / 2} e^{i t r[\eta \cdot \theta(\eta)]} \tilde{R}_{+}^{s}(r \eta, t) e^{-i r \xi \cdot \eta} d \sigma(\eta) d r \\
& +t^{(n-1) / 2} \int_{\epsilon}^{R} \phi(r) r^{(n-3) / 2} \int_{\eta \in S^{*}}|\eta|^{-(n+1) / 2} e^{-i t r[\eta \cdot \theta(\eta)]} \tilde{R}_{-}^{s}(r \eta, t) e^{-i r \xi \cdot \eta} d \sigma(\eta) d r .
\end{aligned}
$$

We will focus on the first term since the second one can be handled alike

$$
I_{t}^{S}(\xi)=t^{(n-1) / 2} \int_{\epsilon}^{R} \phi(r) r^{(n-3) / 2} \int_{\eta \in S^{*}}|\eta|^{-(n+1) / 2} e^{i t r[\eta \cdot \theta(\eta)]} \tilde{R}_{+}^{s}(r \eta, t) e^{-i r \xi \cdot \eta} d \sigma(\eta) d r
$$

and note that due to $(4.4) \eta \cdot \theta(\eta)=1$, and thus

$$
I_{t}^{S}(\xi)=t^{(n-1) / 2} \int_{\epsilon}^{R} \phi(r) r^{(n-3) / 2} e^{i t r} \int_{\eta \in S^{*}}|\eta|^{-(n+1) / 2} \widetilde{R}_{+}^{s}(r \eta, t) e^{-i r \xi \cdot \eta} d \sigma(\eta) d r .
$$

One can use the expression $\xi=\|\xi\|_{a} \theta(\xi)$ and employ stationary phase method to obtain

$$
\begin{aligned}
\int_{\eta \in S^{*}}|\eta|^{-(n+1) / 2} \widetilde{R}_{+}^{s}(r \eta, t) e^{-i r \xi \cdot \eta} d \sigma(\eta)= & \|\xi\|_{a}^{-(n-1) / 2} e^{i r\|\xi\|_{a}[\theta(\xi) \cdot \eta(\theta(\xi))]} P_{+}^{s}(r \xi, t) \\
& +\|\xi\|_{a}^{-(n-1) / 2} e^{-i r\|\xi\|_{a}[\theta(\xi) \cdot \eta(\theta(\xi))]} P_{-}^{s}(r \xi, t),
\end{aligned}
$$

where $P_{ \pm}^{s}$ are smooth functions such that $D_{t}^{\alpha} D_{z}^{\beta} P_{ \pm}^{s}(z, t)=O\left(t^{-s-\alpha}\right)$. Using this expression, we have

$$
\begin{aligned}
I_{t}^{S}(\xi)= & \left(\frac{t}{\|\xi\|_{a}}\right)^{(n-1) / 2} \int_{\epsilon}^{R} \phi(r) r^{(n-3) / 2} e^{i r\left(t-\|\xi\|_{a}\right)} P_{+}^{S}(r \xi, t) d r \\
& +\left(\frac{t}{\|\xi\|_{a}}\right)^{(n-1) / 2} \int_{\epsilon}^{R} \phi(r) r^{(n-3) / 2} e^{-i r\left(t-\|\xi\|_{a}\right)} P_{-}^{s}(r \xi, t) d r .
\end{aligned}
$$


Further integrating by parts the integrals, one can see that for any $N>0$ both integrals are controlled by $\left(C_{N} t^{-S}\right) /\left(1+\left|t-\|\xi\|_{a}\right|\right)^{N}$. As a result, we have

$$
\left|I_{t}^{S}(\xi)\right| \leq\left(\frac{t}{\|\xi\|_{a}}\right)^{(n-1) / 2} \frac{C_{N} t^{-S}}{\left(1+\left|t-\|\xi\|_{a}\right|\right)^{N}} \leq \frac{D_{N} t^{-S}}{\left(1+\left|t-\|\xi\|_{a}\right|\right)^{N-((n-1) / 2)}}
$$

uniformly for $|\xi|>1$ and $t>\delta$. Finally, substituting into (5.10), we obtain (5.7). $\xi \in R^{n}$,

Now, combining Lemmas 5.1 and 5.2, we can claim that, in fact, for $t>\delta$ and any

$$
\left|\widehat{\Theta}_{t}^{s}(\xi)\right| \leq \frac{O(1) t^{-s}}{\left(1+\left|\|\xi\|_{a}-t\right|\right)^{\alpha}}
$$

Lemma 5.3. Let $\Theta_{\lambda}^{s}(x)$ be defined by (5.3). Then, for any $\delta>0$ there is $C_{\delta}>0$ such that

$$
\int_{\delta}^{\infty}\left|\widehat{\Theta}_{t}^{s}(\xi)\right|^{2} d t \leq \frac{C_{\delta}}{(1+|\xi|)^{2 s}}
$$

Proof. As it follows from (5.16),

$$
\int_{\delta}^{\infty}\left|\widehat{\Theta}_{t}^{s}(\xi)\right|^{2} d t \leq O(1) \int_{\delta}^{\infty} \frac{t^{-2 s} d t}{\left(1+\left|\|\xi\|_{a}-t\right|\right)^{2 \alpha}}
$$

where $\alpha>0$ can be chosen arbitrary large. Changing the variables $u=\xi-t$, one has

$$
\begin{aligned}
\int_{\delta}^{\infty} \frac{|t|^{-2 s} d t}{\left(1+\left|\|\xi\|_{a}-t\right|\right)^{2 \alpha}}= & \int_{\delta<\left|\|\xi\|_{a}-u\right|<\|\xi\|_{a} / 2} \frac{\left|\|\xi\|_{a}-u\right|^{-2 s} d t}{(1+|u|)^{2 \alpha}} \\
& +\int_{\max \left(\delta,\|\xi\|_{a} / 2\right)<\|\| \xi \|_{a}-u \mid} \frac{\left|\|\xi\|_{a}-u\right|^{-2 s} d t}{(1+|u|)^{2 \alpha}}
\end{aligned}
$$

It is not difficult to see that for the values $u$ in the first integral $|u|>\left|\|\xi\|_{a}-\right| u-\|\xi\|_{a} \mid>\|\xi\|_{a} / 2$, and thus choosing $\alpha>\max (2 s, 1)$

$$
\int_{\delta<\|\xi\|_{a}-u \mid<\|\xi\|_{a} / 2} \frac{\left|\|\xi\|_{a}-u\right|^{-2 s} d t}{(1+|u|)^{2 \alpha}} \leq \frac{O(1)}{\left(1+\|\xi\|_{a}\right)^{\alpha}} \leq \frac{O(1)}{\left(1+\|\xi\|_{a}\right)^{2 s}} .
$$

Moreover, it is clear that for such $\alpha$

$$
\int_{\delta<\|\xi \xi\|_{a}-u \mid<\|\xi\|_{a} / 2} \frac{\left|\|\xi\|_{a}-u\right|^{-2 s} d t}{(1+|u|)^{2 \alpha}} \leq \frac{O(1)}{\left(1+\|\xi\|_{a}\right)^{2 s}}
$$


Therefore,

$$
\int_{\delta}^{\infty}\left|\widehat{\Theta}_{t}^{s}(\xi)\right|^{2} d t \leq \frac{O(1)}{\left(1+\|\xi\|_{a}\right)^{2 s}} .
$$

Since all norms in $R^{n}$ are equivalent, the lemma is proved.

Lemma 5.4. Let $\Theta_{\lambda}^{s}$ be defined by (5.3). Then, for any $\delta>0$, there is $C_{\delta}$ such that

$$
\int_{\delta}^{\infty}\left|\frac{d}{d t} \widehat{\Theta}_{t}^{s}(\xi)\right|^{2} d t \leq \frac{C_{\delta}}{(1+|\xi|)^{2 s}} .
$$

Proof. For any $t>1$, using the Fubini theorem, one has

$$
\int_{1}^{t} \int \frac{d}{d u} \Theta_{u}^{s}(y) e^{-i \xi y} d y d u=\int e^{-i \xi y} \int_{1}^{t} \frac{d}{d u} \Theta_{u}^{s}(y) d u d y=\widehat{\Theta}_{t}^{s}(\xi)-\widehat{\Theta}_{1}^{s}(\xi),
$$

which implies $(d / d t) \widehat{\Theta}_{t}^{s}(\xi)=\left(\widehat{d / d t)} \Theta_{t}^{s}(\xi)\right.$.

If $s>0$

$$
\begin{aligned}
\frac{d}{d t} \Theta_{t}^{s}(x) & =\phi(x) \frac{d}{d t} \theta_{t}^{s}(x) \\
& =\phi(x) \frac{d}{d t} \int_{0}^{t}\left(1-\frac{u^{2}}{t^{2}}\right) d \theta_{u}(x) \\
& =\frac{2 s \phi(x)}{t} \int_{0}^{t}\left(1-\frac{u^{2}}{t^{2}}\right)^{s-1} \frac{u^{2}}{t^{2}} d \theta_{u}(x) \\
& =\frac{2 s}{t}\left(\Theta_{t}^{s-1}(x)-\Theta_{t}^{s}(x)\right) .
\end{aligned}
$$

Thus, using inequality $(a+b)^{2} \leq 2 a^{2}+2 b^{2}$, one has

$$
\int_{\delta}^{\infty}\left|\frac{d}{d t} \widehat{\Theta}_{t}^{s}(\xi)\right|^{2} d t \leq C \int_{\delta}^{\infty} t^{-2}\left|\widehat{\Theta}_{t}^{s-1}(\xi)\right|^{2} d t+C \int_{\delta}^{\infty} t^{-2}\left|\widehat{\Theta}_{t}^{s}(\xi)\right|^{2} d t
$$

Now, one can use estimate (5.16) to each integral on the right side and complete the proof. 
If $s=0$, then for any $\xi \in R^{n}$,

$$
\begin{aligned}
\left|\frac{d}{d t} \widehat{\Theta}_{t}(\xi)\right| & =\left|\frac{d}{d t} \int_{A(y) \leq t} \widehat{\phi}(\xi+y) d y\right| \\
& =\left|\int_{A(y)=t} \widehat{\phi}(\xi+y) n(y) d \sigma(y)\right| \\
& \leq \frac{O(1)}{\left(1+\left|\|\xi\|_{a}-t\right|\right)^{\alpha}}, \quad \forall \alpha>0 .
\end{aligned}
$$

Using this estimate and the reasoning presented in the previous lemma, we obtain the required estimate.

\section{Proof of Theorem 3.1}

Let $f \in H^{-l} \cap \mathcal{E}^{\prime}$ be such that supp $f \subset \Omega$. For $0<\epsilon<1 / 2$, we set

$$
E_{\epsilon}=\left\{x: 2 \epsilon<\operatorname{dist}(x, \Omega)<(2 \epsilon)^{-1}\right\}
$$

and consider an arbitrary radial function $\phi_{\epsilon} \in C_{0}^{\infty}$ such that

$$
\phi_{\epsilon}(x)= \begin{cases}1, & \frac{3 \epsilon}{2} \leq|x| \leq \frac{1}{\epsilon}+\text { diam } \Omega \\ 0, & |x| \leq \epsilon .\end{cases}
$$

It is clear that to prove the theorem it is sufficient to show that for any $\epsilon>0$, $\lim _{\lambda \rightarrow \infty} E_{\lambda}^{S} f(x)=0$, a.e. $x \in E_{\epsilon}$

In this case, as $x \in E_{\epsilon}$ due to (2.6)

$$
\begin{aligned}
E_{\lambda}^{s} f(x) & =\int \widehat{f}(\xi)\left[\chi_{\Omega}(\cdot) \theta_{\lambda}^{s}(x-\cdot)\right]^{\curlyvee}(-\xi) d \xi \\
& =\int \widehat{f}(\xi)\left[\phi_{\epsilon}(x-\cdot) \theta_{\lambda}^{s}(x-\cdot)\right]^{\curlyvee}(-\xi) d \xi \\
& =\int \widehat{f}(\xi)\left[\phi_{\epsilon} \theta_{\lambda}^{s}\right]^{\uparrow}(\xi) e^{i \xi x} d \xi,
\end{aligned}
$$

or using notation (5.3)

$$
E_{\lambda}^{s} f(x)=\int \widehat{f}(\xi) \widehat{\Theta}_{\lambda}^{s}(\xi) e^{i \xi x} d \xi=\left(\widehat{f}\left(\widehat{\xi)} \widehat{\Theta}_{\lambda}^{s}(\xi)\right)(-x)\right.
$$


Further, we consider maximal operator:

$$
E_{*}^{S} f(x)=\sup _{\lambda>1}\left|E_{\lambda}^{S} f(x)\right|
$$

We recall that to prove a.e. convergence on $E_{\epsilon}$ one can use the standard technique of Banach principle (see, e.g., [19]) according to which it is sufficient to estimate maximal operator on $E_{\epsilon} \subset R^{n} \backslash \operatorname{supp} f$ as

$$
\left\|E_{*}^{S} f(x)\right\|_{L_{2}\left(E_{\epsilon}\right)} \leq C\|f\|_{H^{-l}} .
$$

Let $\gamma(t): \mathbb{R} \rightarrow \mathbb{R}_{+}$be a $C^{\infty}$ function such that

$$
r(t)=\left\{\begin{array}{l}
0, \quad t \leq \frac{1}{3} \\
1, \quad t \geq \frac{2}{3}
\end{array}\right.
$$

If we set $\widetilde{E}_{\lambda}^{s} f(x)=\gamma(\lambda) E_{\lambda}^{s} f(x)$, then by (6.4),

$$
\tilde{E}_{\lambda}^{s} f(x)=\gamma(\lambda)<f(\cdot), \quad \Theta_{\lambda}^{s}(x-\cdot)>=\gamma(\lambda)\left(\widehat{f}\left(\widehat{\xi) \widehat{\Theta}_{\lambda}^{s}}(\xi)\right)(-x) .\right.
$$

According to Sobolev's embedding theorem (see, e.g., [20]) for any $f \in H^{1}\left(R^{1}\right)$,

$$
\|f\|_{L_{\infty}} \leq C\|f\|_{H^{1}} .
$$

Using this fact, we have

$$
E_{*}^{s} f(x) \leq\left\|\tilde{E}_{\lambda}^{s} f(x)\right\|_{L^{\infty}(R)} \leq\left\|\tilde{E}_{\lambda}^{s} f(x)\right\|_{H^{1}(R)}
$$

And, therefore, in order to obtain (6.6), it is sufficient to show that there are constants $C_{1}, C_{2}$ such that the following estimates are true:

$$
\begin{gathered}
\int\left\|\widetilde{E}_{\lambda}^{s} f(x)\right\|_{L_{2}(R)}^{2} d x \leq C_{1}\|f\|_{H^{-l}}, \\
\int\left\|\frac{d}{d \lambda} \widetilde{E}_{\lambda}^{s} f(x)\right\|_{L_{2}(R)}^{2} d x \leq C_{2}\|f\|_{H^{-l}} .
\end{gathered}
$$

First, we note that estimate (5.16) and $f \in H^{-l}$ imply that $\widehat{f} \widehat{\Theta}_{\lambda}^{s} \in L_{2}$ which in turn with (6.8) implies the fact $\widetilde{E}_{\lambda}^{s} f \in L_{2}$. 
Further, using the Plancherel theorem, we have

$$
\begin{aligned}
\iint\left|\widetilde{E}_{\lambda}^{s} f(x)\right|^{2} d \lambda d x & \leq \int r^{2}(\lambda) \int|\widehat{f}(\xi)|^{2}\left|\widehat{\Theta}_{\lambda}^{s}(\xi)\right|^{2} d \xi d \lambda \\
& \leq \int_{1 / 3}^{\infty} r^{2}(t) \int\left(1+|\xi|^{2}\right)^{l}\left|\widehat{\Theta}_{t}^{s}(\xi)\right|^{2}\left(1+|\xi|^{2}\right)^{-l}|\widehat{f}(\xi)|^{2} d \xi d t \\
& \leq \sup _{\xi \in R^{n}}\left(1+|\xi|^{2}\right)^{l} \int_{1 / 3}^{\infty}\left|\widehat{\Theta}_{t}^{s}(\xi)\right|^{2} d t \times\|f\|_{H^{-l}}^{2} \leq C\|f\|_{H^{-l}}^{2}
\end{aligned}
$$

(the last inequality follows from Lemma 5.3).

For the same reason, (6.11) can be proved using Lemmas 5.3 and 5.4:

$$
\begin{aligned}
\iint_{1 / 3}^{\infty}\left|\frac{d}{d t}\left[\gamma(t) \Theta_{t}^{s}\right] * f(x)\right|^{2} d t d x \leq & \left\|r^{\prime 2}(\lambda)\right\|_{\infty} \iint_{1 / 3}^{\infty}\left|\Theta_{t}^{s} * f(x)\right|^{2} d t d x \\
& +\iint_{1 / 3}^{\infty}\left|\frac{d}{d \lambda} \Theta_{\lambda}^{s} * f(x)\right|^{2} d \lambda d x \leq C\|f\|_{H^{-l}}^{2}
\end{aligned}
$$

\section{Acknowledgments}

The authors are thankful to the University Putra Malaysia for the support under RUGS (Grant no. 05-03-11-1450RU). A. Butaev would also like to expresses his gratitude to for the support under IGRF scheme.

\section{References}

[1] V. Ii'in, "On a generalized interpretation of the principle of localization for Fourier series with respect to fundamental systems of functions," Sibirskiŭ Matematičeskiŭ Žurnal, vol. 9, no. 5, pp. 1093-1106, 1968.

[2] P. Sjölin, "Regularity and integrability of spherical means," Monatshefte für Mathematik, vol. 96, no. 4, pp. 277-291, 1983.

[3] A. Carbery and F. Soria, "Almost-everywhere convergence of Fourier integrals for functions in Sobolev spaces, and an $L^{2}$-localisation principle," Revista Matemática Iberoamericana, vol. 4, no. 2, pp. 319-337, 1988.

[4] A. Carbery and F. Soria, "Pointwise Fourier inversion and localisation in $R^{n}$," The Journal of Fourier Analysis and Applications, vol. 3, supplement 1, pp. 847-858, 1997.

[5] A. Bastis, "Generalized localization of Fourier series with respect to the eigenfunctions of the laplace operator in the classes $L_{p}$ classes," Litovskiŭ Matematicheskiu Sbornik, vol. 31, no. 3, pp. 387-405, 1991.

[6] A. Bastis, "The generalized localization principle for an N-fold Fourier integral," Doklady Akademii Nauk SSSR, vol. 278, no. 4, pp. 777-778, 1984.

[7] A. Bastis, "On the generalized localization principle for an $\mathrm{N}$-fold Fourier integral in the classes $L_{p}$," Doklady Akademii Nauk SSSR, vol. 304, no. 3, pp. 526-529, 1989.

[8] R. Ashurov, A. Ahmedov, and A. Rodzi b. Mahmud, "The generalized localization for multiple Fourier integrals," Journal of Mathematical Analysis and Applications, vol. 371, no. 2, pp. 832-841, 2010.

[9] F. J. González Vieli and E. Seifert, "Fourier inversion of distributions supported by a hypersurface," The Journal of Fourier Analysis and Applications, vol. 16, no. 1, pp. 34-51, 2010.

[10] J. Vindas and R. Estrada, "Distributional point values and convergence of Fourier series and integrals," The Journal of Fourier Analysis and Applications, vol. 13, no. 5, pp. 551-576, 2007.

[11] J. Vindas and R. Estrada, "On the order of summability of the Fourier inversion formula," Analysis in Theory and Applications, vol. 26, no. 1, pp. 13-42, 2010. 
[12] J. Vindas and R. Estrada, "On the support of tempered distributions," Proceedings of the Edinburgh Mathematical Society 2, vol. 53, no. 1, pp. 255-270, 2010.

[13] Sh. A. Alimov, "On spectral decompositions of distributions," Doklady Akademii Nauk, vol. 331, no. 6, pp. 661-662, 1993.

[14] Sh. A. Alimov and A. Rakhimov, "On the localization of spectral expansions of distributions," Journal of Differential Equations, vol. 32, no. 6, pp. 792-802, 1996.

[15] Sh. A. Alimov and A. Rakhimov, "On the localization of spectral expansions of distributions in a closed domain," Journal of Differential Equations, vol. 33, no. 1, pp. 80-82, 1997.

[16] Y. Egorov, Linear Differential Equations of Principal Type, Consultants Bureau, New York, NY, USA, 1986.

[17] E. M. Stein and G. Weiss, Introduction to Fourier Analysis on Euclidean Spaces, Princeton University Press, 1971.

[18] M. V. Fedoryuk, The Saddle-Point Method, Moscow, Russia, 1977.

[19] A. Garsia, Topics in Almost Everywhere Convergence, vol. 4 of Lectures in Advanced Mathematics, Markham Publishing Corporation, Chicago, Ill, USA, 1970.

[20] Sh. A. Alimov, R. Ashurov, and A. Pulatov, "Multiple Fourier series and Fourier integrals," in Commutative Harmonic Analysis IV, vol. 42 of Encyclopaedia of Mathematical Sciences, pp. 1-97, Springer, 1992. 


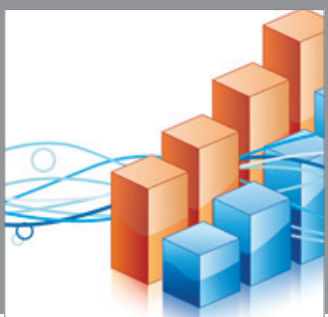

Advances in

Operations Research

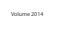

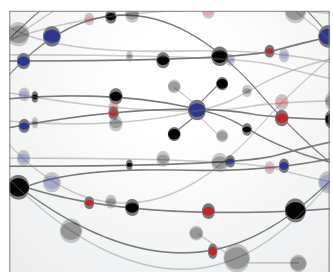

\section{The Scientific} World Journal
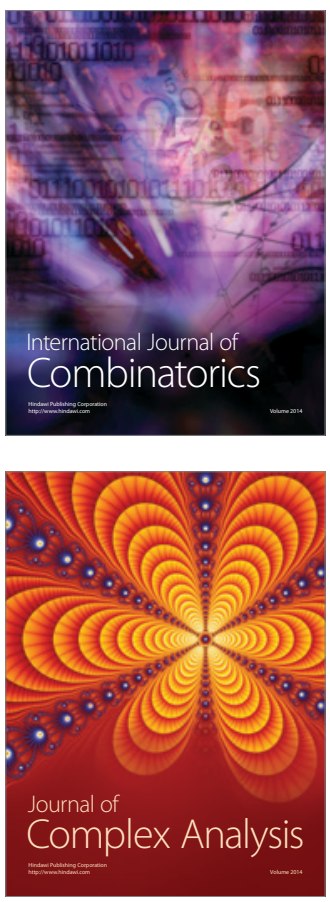

International Journal of

Mathematics and

Mathematical

Sciences
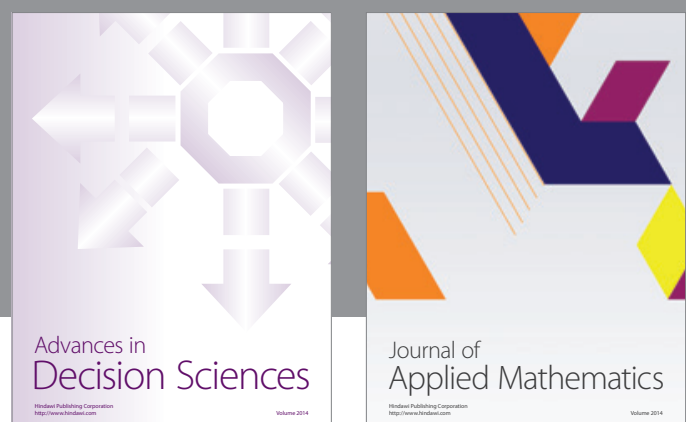

Journal of

Applied Mathematics
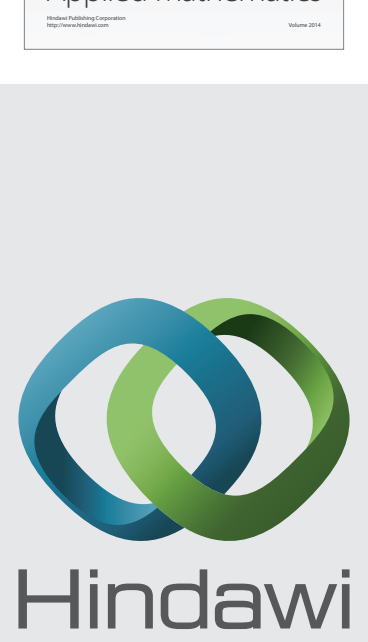

Submit your manuscripts at http://www.hindawi.com
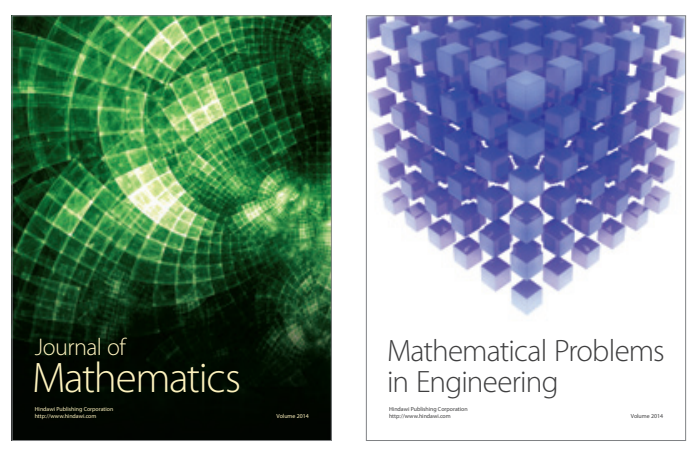

Mathematical Problems in Engineering
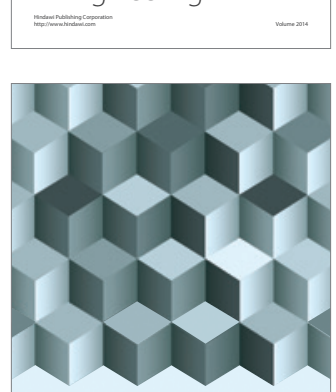

Journal of

Function Spaces
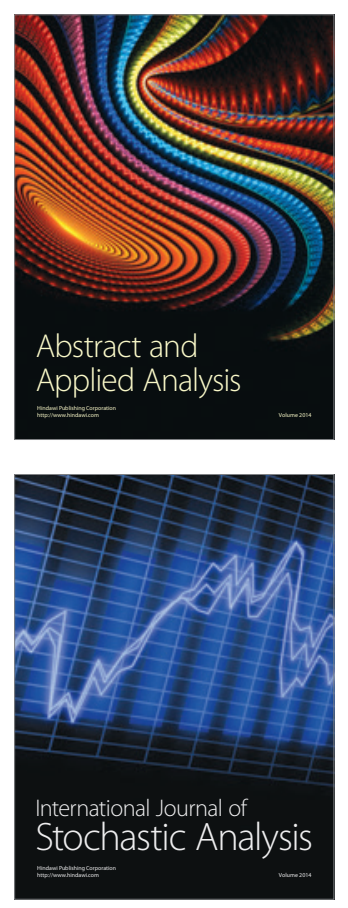

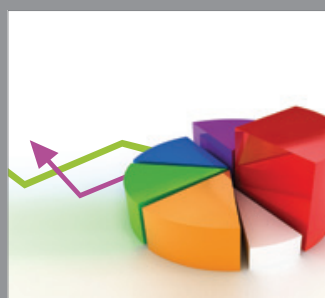

ournal of

Probability and Statistics

Promensencen
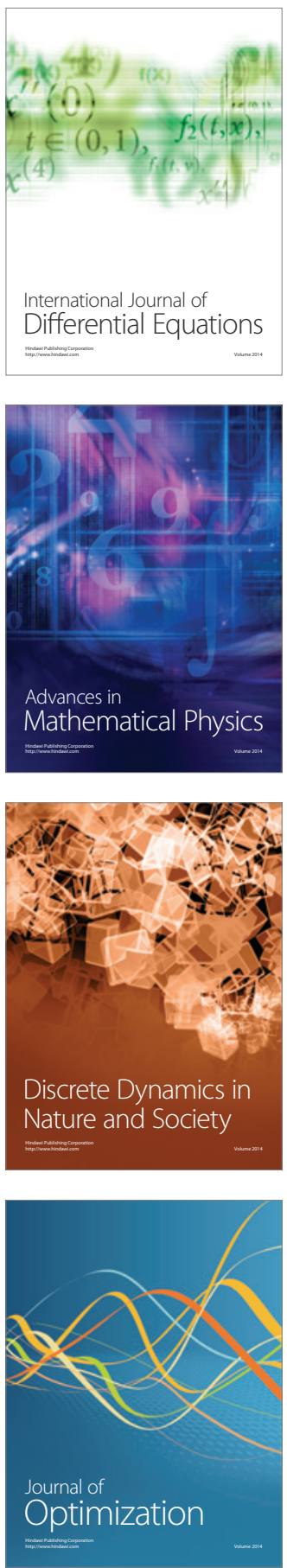